\title{
X-Ray Contrast Media Mechanisms in the Release of Mast Cell Contents: Understanding These Leads to a Treatment for Allergies
}

\author{
Elliott C. Lasser \\ UCSD Emeritus, USA \\ Correspondence should be addressed to Elliott C. Lasser, elasser@ucsd.edu \\ Received 14 May 2011; Revised 15 July 2011; Accepted 16 July 2011 \\ Academic Editor: K. Blaser
}

Copyright () 2011 Elliott C. Lasser. This is an open access article distributed under the Creative Commons Attribution License, which permits unrestricted use, distribution, and reproduction in any medium, provided the original work is properly cited.

\begin{abstract}
A long history of searching for the etiology of X-ray contrast material (CM) reactions has led to the understanding that the CM do not produce anti-CM antigens. Since CM reactions are anaphylactoid in nature, however, a source for mast cell activation was sought. This resulted in the finding that concentrated CM could suppress mast cell activation by attachment to the Fc portion of $\mathrm{IgE}$ and IgG. This is presumed to be a steric hindrance effect. In a study of the effects of CM on BP and a study of the effects of $\mathrm{CM}$ in sensitized rats, it was concluded that less concentrated CM activated mast cells and that this mechanism was best explained by bridging of adjacent IgE molecules via attachment to their Fc segments. The mast cell release of heparin activating the contact system, as well as the release of histamine, is believed to be responsible for CM reactions and allergic diatheses.
\end{abstract}

\section{Contrast Media Molecular Structures}

Currently all X-ray contrast media (CM) for intravascular opacification are tri-iodinated benzene moieties that are fully substituted and occur in either a monomer or a dimer form (Figure 1).

\section{The Contact System in CM Anaphylaxis}

The contact system is made up of three proteins (Figure 2): (1) Factor XII, the protein that initiates activation of the intrinsic coagulation system and can be activated by negative surfaces in the circulation, (2) prekallikrein, and (3) high molecular weight kininogen. Activation of Factor XII converts prekallikrein to kallikrein, which converts high molecular kininogen to bradykinin. Bradykinin has essentially the same physiologic effects as histamine but is significantly more effective on a mole per mole basis. Furthermore, bradykinin, once formed, can metabolize arachidonic acid into vasoactive prostaglandins and leukotrienes, all of which are known to participate in anaphylactic events.

\section{Activation of the Contact System and Negative Surfaces}

The elements mentioned above constitute in outline what is known about some of the important mechanisms that play a role in anaphylaxis and allergy. Now, it is necessary to understand what leads up to these events and what can be done to limit them. Negative surfaces in the circulation can activate the contact system. In the body negative surfaces exist in a number of forms. The activation of factor XII will occur when these surfaces have access to this factor in sufficient concentration. In vitro, we have shown that high molecular weight dextran sulfate can activate the system when the temperature is sufficiently low to inhibit plasma substances that would otherwise impede this activation [1]. More importantly, we have demonstrated that this process proceeds more rapidly in atopic or asthmatic individuals than in individuals without these abnormalities [1]. Asthmatics can be demonstrated to have higher concentrations than do nonasthmatics of heparin/heparan sulfate when these molecules are isolated from their neutralizing substances [2, 3] (Figure 3).

\section{The Role of Heparin-Like Substances}

What are the sources of these heparin-like molecules? While heparan sulfate can be found in a number of tissues, including endothelial linings, the most obvious source of acute supply of heparin-like molecules is the mast cell (Figure 4). When activated, these cells discharge a large number of substances, including heparin, histamine, tryptase, chymase, 


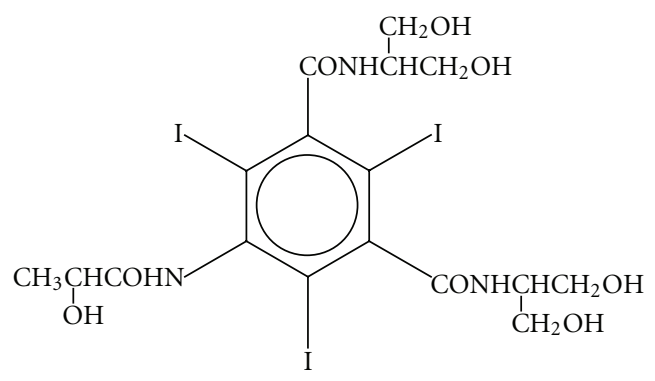

Iopamidol

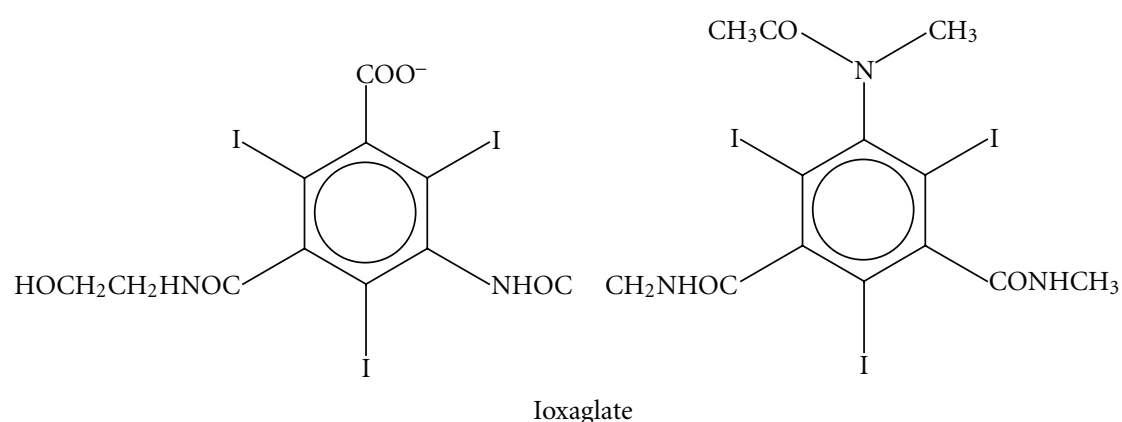

FIgURE 1: Representative current X-ray contrast molecules. Iopamidol is a nonionic monomer. Ioxaglate is an ionic dimer. All current contrast media have iodide atoms on the 2,4 , and 6 positions on the benzene ring.

Contact System

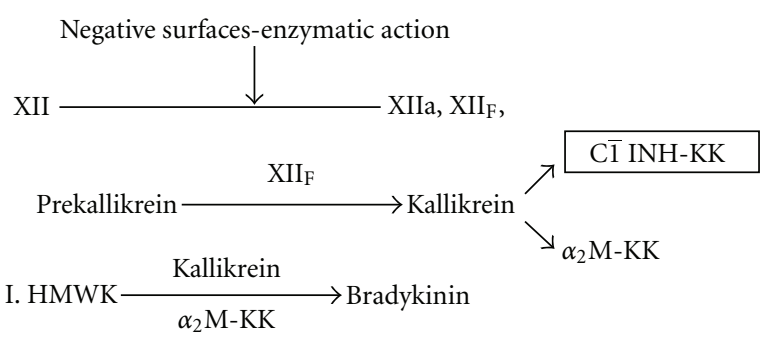

FIGURE 2: The 3 major proteins of the contact system Factor XII, Prekallikrein, and high molecular weight kininogen are depicted. The major role played by the C1 inhibitor is noted. Factor XIIf is a breakdown product of Factor XII.

and ACE and a number of cytokines and chemokines. The in vivo activation of the mast cells, in turn, results when IgE attached to specific receptors on the mast cell surfaces combine with specific antigens in the circulation to produce aggregation of adjacent receptors [4]. (In all our references to the mast cell in this paper, it is understood that basophils in the circulation largely exhibit similar characteristics.) How does heparin/heparan become the negative surface that will activate the contact system in CM reactions? The answer to this lies in putting together two counterintuitive investigations carried out approximately 27 years apart.

\section{The Attachment of CM to IgE and IgG (The Fallacy of Anticipated Data)}

In the first of the counterintuitive experiments mentioned above, dogs underwent 27 injections of iothalamate (Conray 60 , Mallinckrodt) into the pulmonary artery and blood samples were collected from a catheter in the left ventricle. The iothalamate was injected in the same volume $(30 \mathrm{~mL})$ in each animal but at different injection rates $(2 \mathrm{~mL} / \mathrm{sec}$ and $39 \mathrm{~mL} / \mathrm{sec}$ ). The left ventricular samples were assayed for total histamine content over a 5-minute interval. To our surprise, the maximal histamine blood levels were higher in 20 of the 27 injections when the $\mathrm{CM}$ was injected more slowly [6]. In the second of the two experiments, RBC hemagglutination inhibition studies were carried out with several stereotypical CM molecules. The CM used were the same concentrations carried in the commercial vials. (141$320 \mathrm{mgI} / \mathrm{mL}$ ). In the study, several different antigens were used. While all the tested CM interfered with the antibodyantigen reactivity at different concentrations, the ionic and nonionic dimers and nonionic monomers accomplished this at lesser concentrations of $\mathrm{CM}$ than did the ionic monomers [7] (Figure 1). This was counterintuitive, since the interference implied that the $\mathrm{CM}$ attached to the antibodies to the exclusion of the specific antigens to these antibodies. Attachment to the antibody by the $\mathrm{CM}$ was reasoned to be at the basis of IgE activation of mast cells as was the case with all antigens. In the case of true antigens, the best attachment to the immunoglobulin (at Fab) produces the 


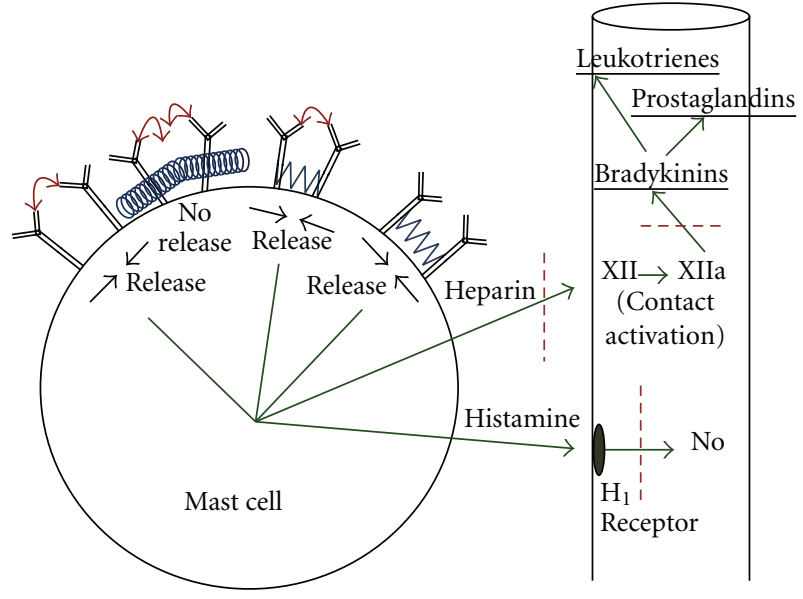

Figure 3: The mast cell dynamics are depicted. Mast cell release occurs when the IgE receptors aggregate and come closer to one another. IgE on the cell is shown to be bridged by antigens (red arrows) binding to the Fab, segment, resulting in mast cell release. Release also occurs in the presence of antigen attachment plus low concentration CM attachment (blue wave forms). Finally, release occurs when there is low CM concentration attachment alone. High concentrations of $\mathrm{CM}$ are thought to act by steric hindrance alone and/or by sterically dislodging bound true antigens. The red dashes represent points of possible corticosteroid inhibitory effect.

W.W-homodendrum spores arterial samples

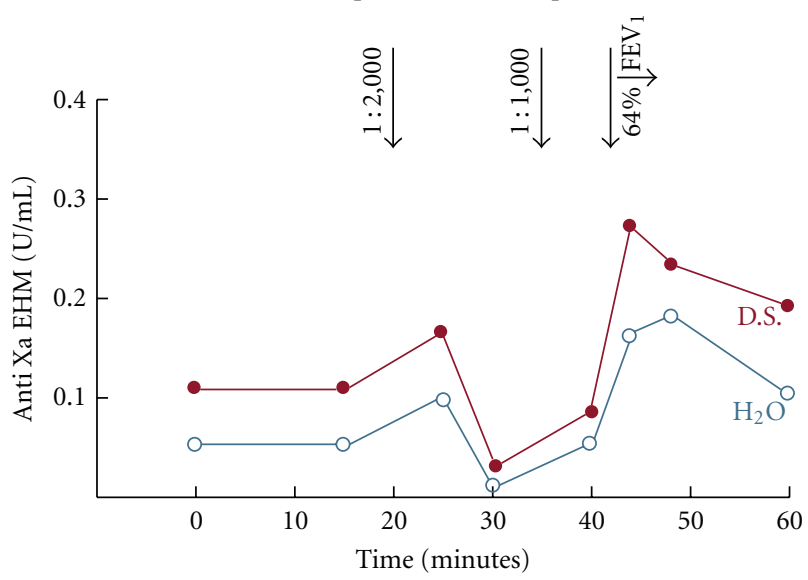

FIGURE 4: In this study, 6 patients were subjected to inhalational challenge with antigens to which they were known to be sensitive [5]. Simultaneous samples of blood were drawn from the brachial artery and from the antecubital vein. A representative patient is shown here. EHM is endogenous heparin-like material, measured with a functional anti-Xa assay after removing neutralizing substances from the heparin by adding low molecular weight dextran sulfate. After the 1:1000 spore challenge, there is an immediate rise in both the EHM material produced by exposing plasma to low molecular weight dextran (red line) and the material with heparin neutralizing substances intact when water, rather than dextran sulfate, was added to the plasma (blue line). A concurrent fall in FEV1 occurs along with the rise in heparin levels when the $1: 1,000$ antigen was inhaled. Similar findings occurred in 3 of the 6 patients.

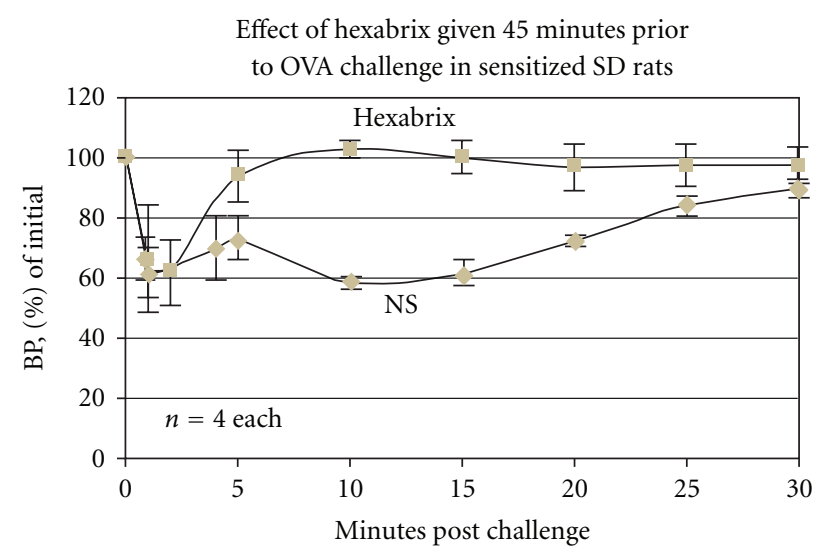

Figure 5: Rats sensitized to ovalbumin and then challenged with ovalbumin, displayed significantly different BP patterns in the presence of either Hexabrix or normal saline. With Hexabrix, whether given 45 minutes earlier, at the time of antigen challenge, or at the nadir of BP levels, there was an abrupt upward swing in $\mathrm{BP}$ levels in contradistinction to the prolonged fall in the presence of the saline.

most activation of the mast cell [7]. In the hemagglutination inhibition study, however, the best attachment was to the $\mathrm{CM}$ most unlikely to clinically activate the IgE on mast cells. Then, remembering the puzzling histamine release results in the study done 27 years earlier, a realization dawned on us. In the earlier experiment, we were looking at a SUPPRESSION of IgE activated mast cell activity by the faster injection, not the expected ACTIVATION! The target tissue in this experiment was the lung, and the histamine release from pulmonary mast cells was detected in the heart so that the release was modulated by a high concentration of CM not usually available in other peripheral target tissues.

We also realized that the $\mathrm{CM}$ must attach to the $\mathrm{Fc}$ (constant) portion of the immunoglobulins and not the Fab (variable) portion where most antigens attached, since the attachment occurred with multiple antigens and was therefore nonspecific. In a limited subsection of this study, we found that CM could apparently detach specific antigens if the antigen-antibody complex were shaken for a few minutes before the $\mathrm{CM}$ was added.

\section{CM Attachment to the Immunoglobulins}

A number of studies to substantiate the foregoing were then carried out. The first was that the CM, attaching to the constant portion of the immunoglobulin, then had the potential to interfere with attachment of specific antigens to the variable part of the immunoglobulin. This was borne out in follow-up studies [8]. Further, we must come to terms with the fact that in vivo and in vitro, CM, in fact, could activate as well as suppress mast cell activity. To accommodate this fact, it was necessary to postulate that at lesser concentrations attachment of CM to Fc portions of immunoglobulins may activate IgE and lead to the adverse reactions sometimes associated with intravascular CM injections, while at higher concentrations, CM will inhibit activation (Figure 3). On a 
theoretical basis, this now seemed reasonable, since all the $\mathrm{CM}$ are known to show some degree of aggregation at higher concentrations (all the CM have a calculated osmolality that is higher than their experimental values) [9]. It seems probable that at the higher concentrations the CM aggregation produces a steric hindrance effect that limits the necessary receptor aggregation for mast cell activation. The other finding that should be emphasized is that CM are completely nonspecific; that is, they can interfere with any antigen binding to $\operatorname{IgG}$ or $\operatorname{IgE}$, and thus merit consideration in any circumstance where CM are present and antigenantibody reactions take place.

\section{Where and Why Do the CM Bind to IgE?}

In our studies that showed that all of the CM could attach to the Fc portion of immunoglobulins and that the ionic and nonionic dimers bound at lesser concentrations than the monomers, it must be assumed that it is the iodine atoms on the benzene rings that are mainly responsible for the binding to the Fc segments (Figures 1 and 3 ). This assumption is justified, since the iodines are the only commonality amongst the various $\mathrm{CM}$, there are more iodines available on the dimer than on the monomer, and less molar concentration of the dimer is necessary to inhibit antigen attachment on the RBC hemagglutination assay. Finally, it should be noted that iodine has been shown to bind to some carbohydrate structures although this may be specific for amylose and elementary iodine [10]. Central carbohydrates are present on the Fc structure of these immunoglobulins, and while we have no proof, these would seem to be the most obvious site for these bindings.

\section{Further Data in Proof}

To substantiate the considerations put forward in the above paragraphs, a number of studies were done: PCA (passive cutaneous anaphylaxis) rat study done with an ionic monomer, meglumine/sodium diatrizoate (Angiovist 370, Bracco Diagnostics), showed an inhibition of immune mediated permeability in rough proportion to the CM used [8]. In a study of BP changes in rats injected IV with various CM, the $\mathrm{CM}$ demonstrating the poorest (least) binding to antibodies in the RBC hemagglutination inhibition tests (the ionic monomers) showed a drop in BP on injection proportionate to the volumes injected [8]. The CM with better binding (the ionic and nonionic dimers) showed an increase in B.P. on injection, again in proportion to the volumes injected [8]. Injections of 2 strains of rats with diphenhydramine, (Benadryl, Mc Neil-PPC) at a dose of $5 \mathrm{mg} / \mathrm{kg}$ also produced a rise in $\mathrm{BP}$ as did injections of L-Name, an inhibitor of nitric oxide [8]. Sensitized rats challenged with the sensitizing antigen and then injected with either a dimer CM or saline showed that the saline injection continued the fall in BP occasioned by the challenge, while the CM produced an upward change in the BP [8]. (Figure 5). These studies were interpreted to show that the CM with better (increased) binding to the Fc portions of IgE inhibited mast cell activity, and thereby diminished the resultant basal blood histamine and NO levels producing higher BP.

\section{Test of the Concepts in Humans}

To test our assumptions that the construct that we had put together would have a role in human allergy, we arranged for a 20-person study of allergic rhinitis with ioxaglate 320 (Hexabrix, Guerbet) versus placebo, in individuals with tested sensitivities to the applied antigens [11]. This was a double-blinded crossover study, and the antigen was applied on pads to the nasal mucosa 20 minutes after 2 drops of ioxaglate 320 or saline were dropped into the nostril. In these studies, the patients score the usual symptoms of nasal allergy on a $1+$ to $3+$ scale. Without going into details, the application of the CM produced fewer symptoms than the placebo in all evaluations. Significant differences ( $t$-test) were found for "sneezing" $(P=0.018)$ and "runny nose" $(P=$ $0.048)$ and the combination of "sneezing", "runny nose" and "itching" was $(P=0.06)$.

\section{Application versus Circulating Anti-IgE}

All of these findings indicate that the CM when applied topically act on peripheral targets in the same fashion as do injections of the monoclonal humanized anti-IgE antibody omalizumab (Xolair, Genentech/Novartis) [12]. Both bind to the Fc portion of antibodies. The action of the CM, however, is immediate and probably acts on both mast cells seated IgE as well as IgE in the local circulation and interstitium, since the effects are abrupt, while the production and action of the monoclonal antibody takes place only over time and is said to be precluded from activity on IgE already seated on mast cells. Although we have no proof of this, it seems likely that CM attachment to local interstitial IgE, like Xolair, will down regulate the density of the IgE receptor on regional mast cells if applied over time.

Since "application" of the CM easily provides the dose and concentration necessary (about 1/250 of common intravascular dosages) for inhibition of all local mast cell activating processes, it is significant to consider the various possible clinical applications which include the nose (allergic rhinitis), the eye (allergic conjunctivitis), the bronchi (asthma), the skin (atopic dermatitis), the esophagus (eosinophilic esophagitis), and possibly intra-articular spaces, the colon, and any other space open to installation or catheterization in which the mast cells are believed to play a significant role.

\section{Technical Note}

As an additional note, it must be realized that the potential of $\mathrm{CM}$ to inhibit competing antigens for attachment to antibodies makes it important to be sure that in the presence of CM in the circulation, the results of assays that depend on correct antigen-antibody dynamics, such as ELISA, are regarded with suspicion. This was first noted in a Japanese study, where tumor antigens were assayed and found to be less than expected quantitatively. It was noted by the authors that this occurred in several assays, where the patients had recently received intravascular $\mathrm{CM}$ and the association was made, but the explanation for the association was not forthcoming [5]. 


\section{Conclusions}

We are aware now that $\mathrm{CM}$ have potential pharmacologic applications beyond conventional opacification in X-ray studies. They are unique for small molecules in that they will inhibit ANY antigen-antibody reaction. Principles gleaned from research in CM physiology have suggested to us a new and completely unique approach to the treatment of hypersensitivity reactions. The abrupt inhibition of release of heparin and other mast cell products by sufficiently concentrated CM applied topically, and the profound inhibition of contact system activation is the key to this.

While our research to date highlights CM inhibition of mast cells and symptoms of acute allergic rhinitis, we are aware that other cellular effectors including dendritic cells and monocytes, which are essential in the pathogenesis of chronic allergic rhinitis, atopic dermatitis, and asthma, also respond to upregulated IgE receptors that potentially may be inhibited in a similar fashion. In this regard, we have had encouraging results in treating a mouse model of atopic eczema, and expect similar results in humans.

It is also possible that $\mathrm{CM}$ injected intravascularly in sufficient concentrations to bind to $\operatorname{IgE}$ in the circulation or on mast cells with the potential to compete with sensitizing antigens can be considered in the therapy of severe anaphylaxis. We have already demonstrated that this is possible in rats (Figure 5), and there is some evidence that the injection of epinephrine in these circumstances is not always accomplishing what is expected [13]. This approach, however, would entail considerable additional research, since suboptimal concentrations could activate mast cell anaphylaxis.

\section{Acknowledgments}

The author wishes to extend his gratitude to a number of people that actively participated in the creative work done over the years: Joseph Lang, Ph.D., Ronald Simon M.D., Sandra Lyon. B.S., and Geraldine Lamkin B.S. He also want to thank his son, Kenneth Lasser M.D., for his helpful ideas and assistance in preparing the paper. The author has received a milestone payment from Asphelia Biopharmaceuticals.

\section{References}

[1] E. C. Lasser, J. H. Lang, S. G. Lyon, A. E. Hamblin, and M. M. Howard, "Prekallikrein-Kallikrein conversion rate as a predictor of contrast material catastrophies," Radiology, vol. 140, no. 1, pp. 11-15, 1981.

[2] E. C. Lasser, R. A. Simon, S. G. Lyon, A. E. Hamblin, and R. Stein, "Heparin-like anticoagulants in asthma," Allergy, vol. 42, no. 8, pp. 619-625, 1987.

[3] S. G. Lyon, E. C. Lasser, and R. Stein, "Modification of an amidolytic heparin assay to express protein-bound heparin," Thrombosis and Haemostasis, vol. 58, no. 3, pp. 884-887, 1987.

[4] R. Schweitzer-Stenner and I. Pecht, "Cutting edge: death of a dogma or enforcing the artificial: monomeric IgE binding may initiate mast cell response by inducing its receptor aggregation," Journal of Immunology, vol. 174, no. 8, pp. 44614464, 2005.
[5] N. Watanabe, N. Oriuchi, T. Higuchi et al., "In vitro effect of contrast agents during immunoradiometric assay for tumourassociated antigens," Nuclear Medicine Communications, vol. 19, no. 1, pp. 63-70, 1998.

[6] E. C. Lasser, "The multipotential pseudoantigenicity of x-ray contrast media: pseudoantigen excess may downregulate the release of hypotensive mediators," International Archives of Allergy and Immunology, vol. 123, no. 4, pp. 282-290, 2000.

[7] E. C. Lasser and G. E. Lamkin, "Can contrast media act as "pseudoantigens"?” Academic Radiology, vol. 5, pp. S95-S106, 1998.

[8] E. C. Lasser and M. S. Lamkin, "Mechanisms of blood pressure change after bolus injections of x-ray contrast media," Academic Radiology, vol. 9, supplement 1, pp. S72-S75, 2002.

[9] W. Krause, H. Miklautz, U. Kollenkirchen, and G. Heimann, "Physicochemical parameters of x-ray contrast media," Investigative Radiology, vol. 29, no. 1, pp. 72-80, 1994.

[10] C. L. Cronan and F. W. Schneider, "Cooperativity and composition of the linear amylose-iodine-iodide complex," Journal of Physical Chemistry, vol. 73, no. 11, pp. 3990-4004, 1969.

[11] Carried out under the supervision of Dr. Robert Naclerio and associates at the University of Chicago Medical School, 2010 with the approval of the institutional review board.

[12] R. C. Strunk and G. R. Bloomberg, "Omalizumab for asthma," The New England Journal of Medicine, vol. 354, no. 25, pp. 2689-2695, 2006.

[13] F. E. R. Simons, "Epinephrine (adrenaline) in the first-aid, out-of-hospital treatment of anaphylaxis," Novartis Foundation Symposium, vol. 257, pp. 228-243, 2004. 


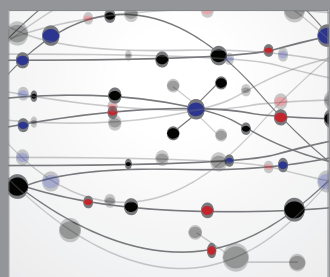

The Scientific World Journal
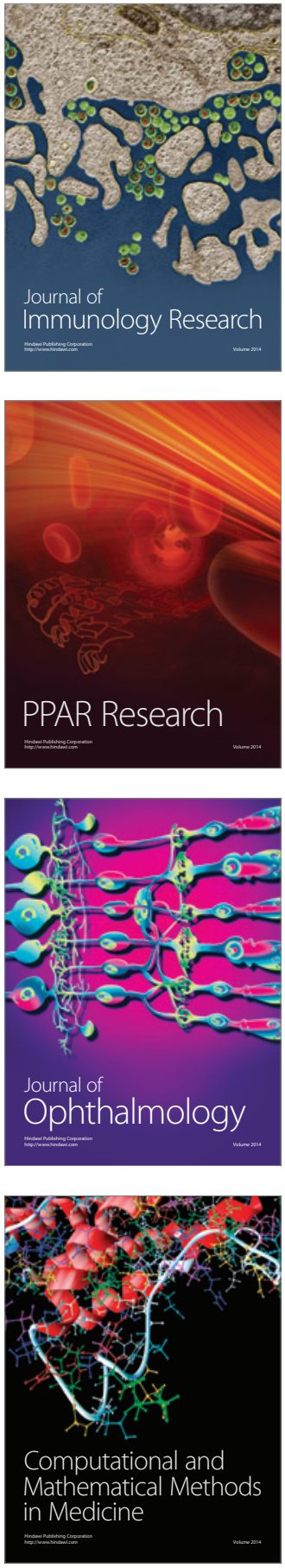

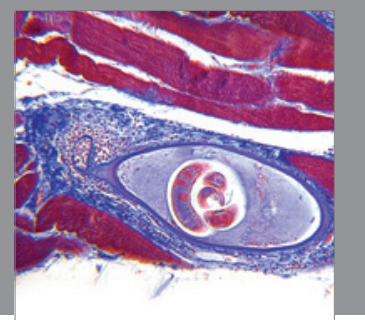

Gastroenterology

Research and Practice
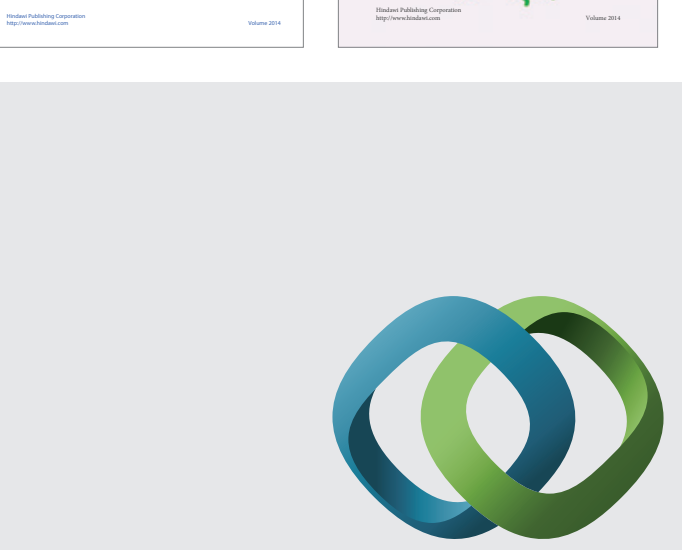

\section{Hindawi}

Submit your manuscripts at

http://www.hindawi.com
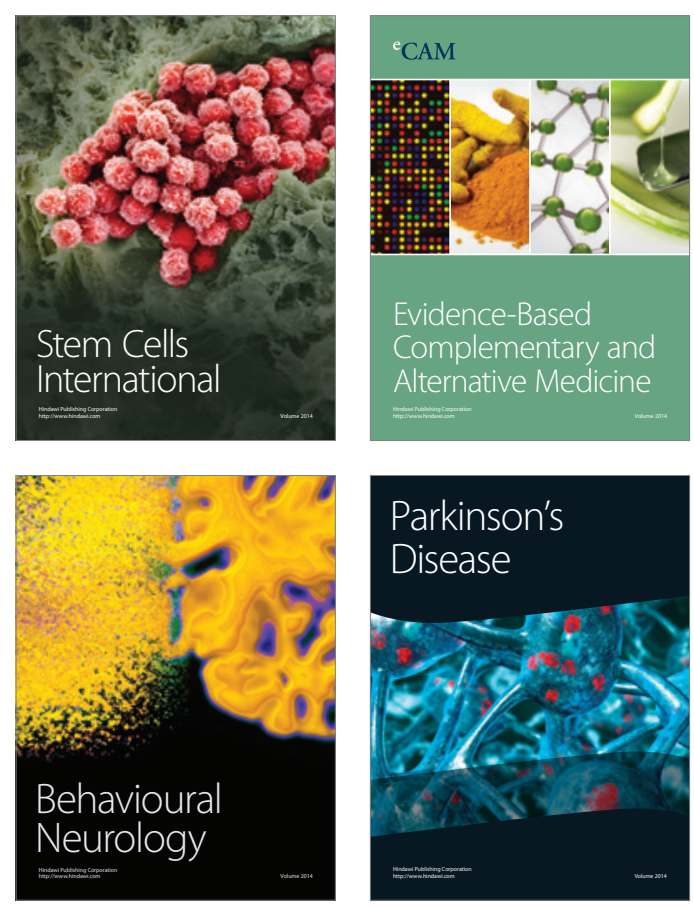

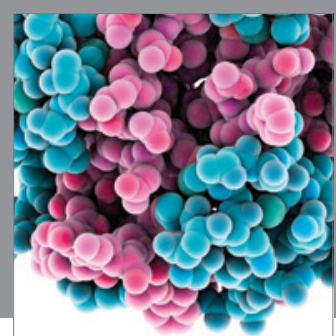

Journal of
Diabetes Research

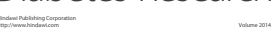

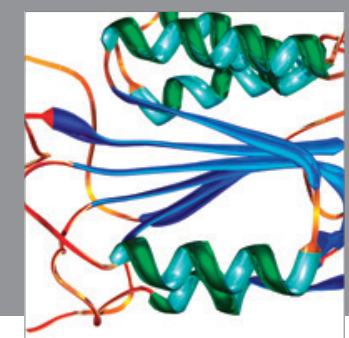

Disease Markers
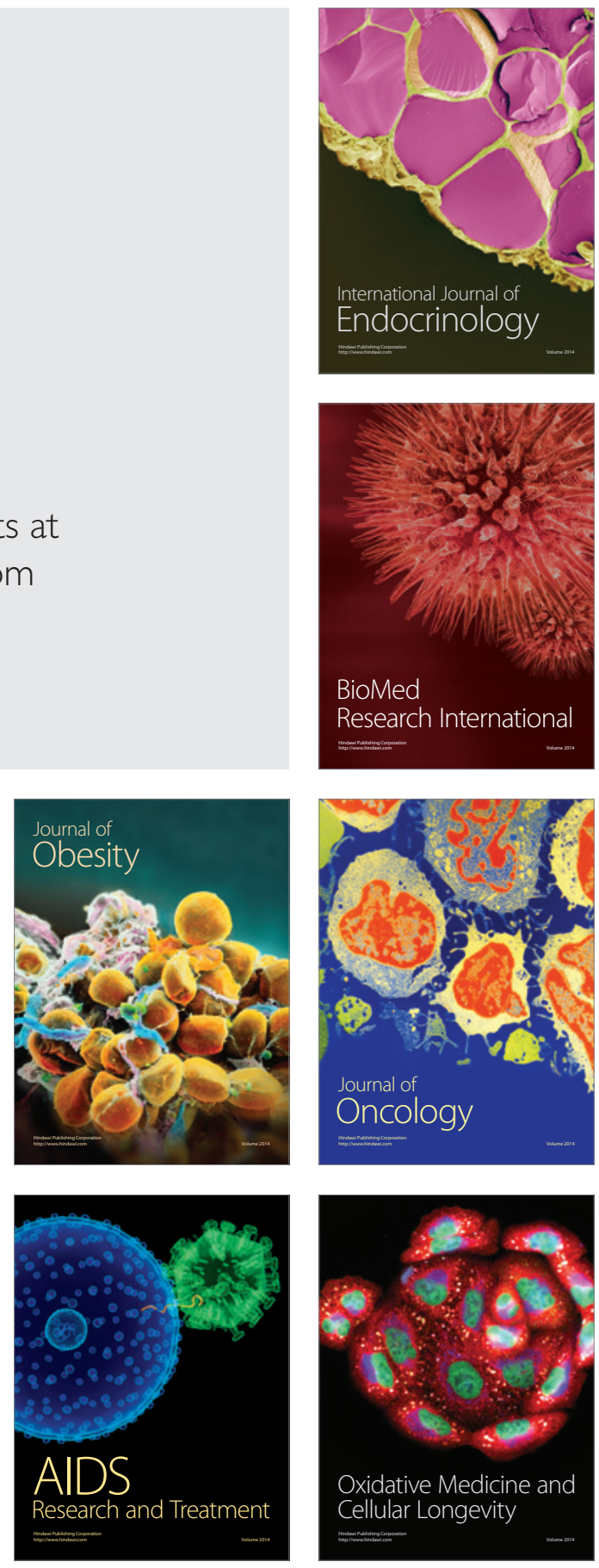